\title{
MEDIACIÓN SOCIAL CON GITANOS EN VILLENA (ALICANTE)'
}

\author{
JOSÉ CABANES HERNÁNDEZ
}

Sociólogo. Servicios Sociales del Ayuntamiento de Villena (Alicante)

I. DESCRIPCIÓN DE LA UBICACIÓN DE LAS FAMILIAS GITANAS DE VILLENA

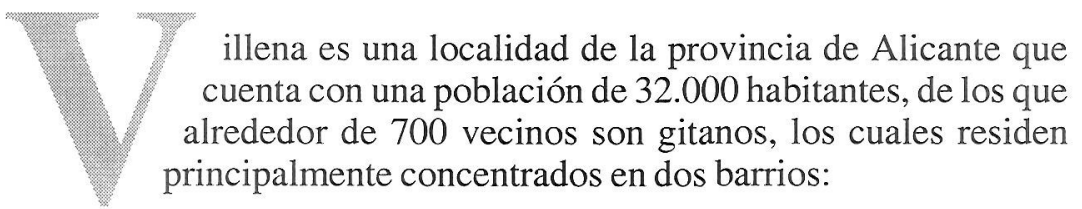

- Barrio del Rabal: es el casco histórico del municipio, donde se encuentra situado el castillo, en cuya parte alta existía un gran número de cuevas excavadas en el monte donde residían muchas familias gitanas, aunque no sólo gitanas. Actualmente nadie vive en cuevas propiamente dichas, aunque muchas de las casas tienen alguna habitación excavada en la roca. Muchas de las viviendas se encuentran deterioradas, y no todas reúnen unas mínimas condiciones de habitabilidad. Las relaciones vecinales son bastante tensas, tanto interétnicas como intraétnicas, en lo cual influye tanto el alto índice de heroinómanos residentes en el barrio, como el hecho de que se concentre allí todo el tráfico de heroína del municipio. La situación laboral es precaria, con empleos temporales y poco cualificados, lo que supone una economía de subsistencia. Todos los niños de este barrio se encuentran escolarizados en el C.P. Ruperto Chapí (un colegio donde el 12\% de los alumnos son gitanos).

- Barrio San Francisco: se construyó en los años 60 en el extrarradio del municipio (se encuentra a $1,5 \mathrm{~km}$. del mismo, sin que exista continuo urbano) para reubicar a la población que vivía en las cuevas. En el barrio hay viviendas en altura y casas unifamiliares, siendo alrededor del $50 \%$ de los vecinos gitanos. Las relaciones

Ponencia en el IV SEMINARIO DE FORMACIÓN: MEDIACIÓN Y EDUCACIÓN INTERCULTURAL, organizado por la Federación de Asociaciones Gitanas Extremeñas y el Instituto Municipal de Servicios Sociales del Ayuntamiento de Badajoz. Badajoz, 24 al 27 de julio de 2000 . 
interétnicas no son malas. Existen problemas de desempleo y trabajos precarios, así como mucha economía sumergida del sector zapatero y juguetero. La mayoría de los niños gitanos están escolarizados en el C.P. Sta. Teresa, situado en dicho barrio, si bien la mayoría de los niños no gitanos están escolarizados en otros centros escolares del municipio (el C.P. Sta. Teresa cuenta con un $80 \%$ de alumnos gitanos).

\section{LA PLAZA DE MONITOR PARA LA POBLACIÓN GITANA}

Mi trabajo depende del departamento de Servicios Sociales, con el que me coordino, pero que no se encuentra situado físicamente dentro de ninguno de los dos barrios con población gitana, por lo que sólo estoy en Servicios Sociales las últimas horas de la mañana y en las reuniones de equipo.

Cuando yo comencé a trabajar como monitor hace dos años y medio, el trabajo que tenía que desarrollar no estaba diseñado, dado que yo era la tercera persona que pasaba por esa plaza en dos años, y cada una de las dos anteriores había hecho cosas distintas. Me planteé dos cosas, lo que quería hacer y lo que no estaba dispuesto a hacer:

- Lo que quería hacer: centrar mi trabajo en el aspecto escolar, rechazando por tanto el convertirme en un cajón desastre que llevase temas de vivienda, empleo, etc.

- Lo que no estaba dispuesto: a ser el "informante clave" de las trabajadoras sociales sobre asuntos de familias gitanas (lo que no quiere decir que no me coordine con los Servicios Sociales).

No quería tener nada que ver en el tema de ayudas económicas a familias. En el sentido que las familias no me tomasen como la puerta de entrada para obtener ayudas de las trabajadoras sociales, ya que en ese caso se establece una relación viciada. De esta forma, las familias tienen claro que aunque trabajo para los Servicios Sociales, no depende de mí el que les den una ayuda económica, o les cedan una vivienda, sino que "el Pepe" sólo lleva el tema de "los nenes" (colegio, actividades extraescolares, etc.). Eso hace que la relación sea más sincera, que no exista esa relación de poder entre quien te puede dar algo, y quien ha de conseguirlo.

\section{OBJETIVO DEL TRABAJO}

El objetivo general que yo me marco es conseguir la escolari- 
zación "normalizada" de todos los alumnos gitanos, entendiendo por normalizada el que tengan las mismas probabilidades de éxito o fracaso escolar que el resto de compañeros.

Evidentemente, es un objetivo a muy largo plazo, pero tiene una diferencia fundamental frente al típico objetivo del absentismo escolar, que tanto preocupa a la Administración. Es que no basta con que los niños vayan al colegio, sino que además hay que trabajar para que esa escolaridad sea fructífera. Eso supone que no sólo hay que trabajar con las familias de los niños absentistas, sino también con las de los que acuden con normalidad, con los profesores de estos niños, y con cualquier persona o institución que pueda favorecer dicho éxito escolar.

\section{LÍNEAS DE TRABAJO A PARTIR DE ESE OBJETIVO}

Para potenciar esa "escolarización normalizada", yo me he marcado distintos objetivos, o líneas de trabajo, que me sirven para no perder el norte en el día a día, e ir consiguiendo pequeños logros que vayan en esa dirección. Estas líneas son:

1. Conseguir la escolarización temprana, a ser posible desde la guardería, pero en cualquier caso, desde la educación infantil de 3 años. Aunque en infantil la enseñanza no es obligatoria, es muy importante trabajar con las familias y las maestras el absentismo en esos primeros años, pues está demostrado que los niños que faltan mucho a estas edades tienen muchas más posibilidades de fracaso escolar.

2. Reducir el absentismo escolar. Digo reducir porque considero que es imposible su erradicación, dado que la situación de precariedad económica de muchas familias hace inevitable cierto tipo de faltas, al tiempo que la visión de las familias gitanas con respecto al absentismo no es la misma que la de la institución escolar. En este sentido, me comentaba una madre gitana hace un par de veranos: "Pepico, tanto venir a verme porque la nena faltaba algunos días, y ahora los payos qué, todo el verano sin dar clase".

3. Retrasar el abandono escolar. Intentar que los niños y niñas gitanas dejen la escuela lo más tarde posible. Hasta hace pocos años, era normal que la mayoría de los menores gitanos dejasen la escuela entre $5^{\circ}$ y $6^{\circ}$ de primaria, pero afortunadamente eso está cambiando. En Villena el primer ciclo de la ESO $\left(1^{\circ}\right.$ y $2^{\circ}$ de ESO) sigue impartiéndose en los colegios de primaria, y en $3^{\circ}$ de ESO pasan al Instituto. Mi objetivo, por ahora, es que nadie deje el colegio antes de terminar $2^{\circ}$ de ESO y que, como mínimo, haga la matrícula en el 
Instituto. Esto puede parecer un objetivo muy alto o muy bajo, según se mire:

- Puede parecer alto porque en $1^{\circ}$ y $2^{\circ}$ de ESO algunos alumnos gitanos tienen un desfase escolar muy grande y apenas hacen ya nada en el colegio y además algunos colegios prefieren que dejen de ir para que no les den problemas, y en ocasiones las familias los necesitan para que les ayuden laboralmente.

- Y puede parecer bajo porque la Ley marca como obligatoria la escolarización hasta los 16 años. Y este objetivo supone dar por hecho que pueden dejar el colegio antes.

Desde mi punto de vista, creo que los chavales, si quieren dejar el colegio lo van a dejar por muchas leyes que hayan, y si el listón está bajo es más fácil convencerlos para que aguanten uno o dos años ( $2^{\circ}$ de ESO). El que nadie lo deje antes de $2^{\circ}$ de ESO permite que los alumnos que van bien no se contagien del abandono escolar de los que tienen un desfase escolar muy fuerte, y hace que los que vienen detrás no se hagan planteamientos del tipo: "yo en $6^{\circ}$ me lo dejo, porque mi hermano también se lo dejó en $6^{\circ}$ ", sino que tengan claro que hay una edad mínima. El conseguir que todos ellos se matriculen, como mínimo, en el Instituto es una forma de forzar a que se familiaricen con el centro, y que vayan unos días, y confiar en que les resulte agradable y que aguanten allí. Creo que la tradición de gitanos en el Instituto es todavía anecdótica, y que hay que ir sentando bases para que los niños y las familias lo vean con normalidad.

4. Diversificar a los alumnos gitanos en el mayor número de colegios posible (tanto públicos como concertados). Considero que la concentración de muchos alumnos gitanos en un mismo centro conlleva la ghetización del mismo, y ello tiene una repercusión directa en el menor rendimiento académico. Problemas de la concentración:

- Los alumnos gitanos se relacionan casi exclusivamente entre gitanos, con lo que no tienen la oportunidad de conocer a otros niños, y mejorar sus relaciones sociales.

- Los alumnos payos optan por colegios donde no haya gitanos, con lo que nunca pierden el miedo a lo desconocido, y no tienen ocasión de superar sus prejuicios sobre los gitanos.

- Los profesores, si pueden, optan por colegios donde no haya muchos gitanos, con lo que los colegios con concentración de alumnos gitanos cambian mucho de profesorado, siendo en muchos casos profesores sin experiencia laboral (en el colegio Sta. Teresa de Villena, este curso puede darse el caso de que cambie el 
90\% del profesorado).

- Es más fácil convencer a los maestros para que se vuelquen en el objetivo de conseguir buenos resultados académicos de los niños gitanos cuando el número de éstos es bajo. Mientras que en lugares donde hay muchos alumnos, los profesores suelen desmoralizarse o relajarse, y marcar objetivos académicos muy bajos.

El objetivo de diversificar a los alumnos gitanos en el mayor número de colegios posible es algo que se debe favorecer, pero que necesariamente pasa por que sea la familia la que tome esa decisión. La mayoría de las familias gitanas prefieren Ilevar a sus hijos al colegio más cercano a su casa, pero algunas familias piden expresamente conseguir plaza en un colegio donde sus hijos vayan a aprender más, o donde no haya tantos gitanos (normalmente para evitar conflictos con otras familias), y es en esos casos donde hay que conseguir matrícula en otro colegio, cosa que no suele ser fácil.

5. Reducir el fracaso escolar. El fracaso escolar está motivado en muchos casos por el absentismo, y en ese sentido, cuanto menos absentismo haya menos fracaso escolar. Pero también se produce a la inversa, que niños que nunca han sido absentistas fracasan escolarmente, y a partir de ahí se aburren y faltan al colegio. En este aspecto influyen muchas variables, desde la motivación del alumno, la importancia que la familia dé a la escuela, las tareas escolares en casa, la motivación de los profesores, etc. Lo cierto es que muchos alumnos gitanos empiezan a quedarse descolgados en $3^{\circ} \circ 4^{\circ}$ de primaria, y que si no se puede conseguir el que hagan toda su escolaridad con buenas notas, al menos sí se debe intentar que el descuelgue sea lo más tarde posible. En este sentido, me he planteado en este último curso, que si bien tengo que trabajar con todos los niños gitanos, el prestar una mayor atención en aquéllos que potencialmente pueden obtener buenos resultados, con el fin de que no pierdan el ritmo, y al tiempo sirvan de referente para otros compañeros gitanos, y para los profesores (que no siempre es fácil hacerles ver que los niños gitanos también pueden aprobar). En cualquier caso, el concepto de fracaso escolar es muchas veces diferente entre familias y colegios, porque lo que muchos padres gitanos esperan de la escuela (que aprendan a leer, escribir, y las cuatro reglas matemáticas), suele llegar a conseguirse, con lo que cuando dejan el colegio el profesor puede pensar que ha fracasado escolarmente, y la familia todo lo contrario.

6. Fomentar la relación entre familias y colegios, con el fin de que colaboren en la marcha escolar de los niños gitanos. La escuela y las familias siguen siendo dos mundos demasiado alejados, y en 
ocasiones contrapuestos, lo que no favorece la escolarización de los niños gitanos. Muchas familias desconocen el funcionamiento del colegio, e incluso desconfían del trabajo de los maestros, y lo mismo suele pasar a la inversa, y la relación se perpetúa en el tiempo sin que nadie dé el primer paso.

7. Mejorar la formación del profesorado con respecto al conocimiento cultural del pueblo gitano, de la realidad social de éste en Villena, y de las posibles estrategias escolares para mejorar su rendimiento académico. Esto se puede hacer de dos formas:

- Cursos o seminarios específicos sobre el tema.

- La formación continua, basada en el día a día, hablando con los distintos profesores y explicándoles cuestiones puntuales.

Yo confiaba mucho en la primera opción, pero la experiencia del curso pasado, en el que organicé un seminario con ponencias muy interesantes (desde mi punto de vista, y así lo comentaron también muchos profesores), donde participaron 140 profesores, me hacía pensar que éste sería un curso mucho más fácil en el trabajo cotidiano con los maestros. Sin embargo, el resultado no ha sido tan positivo, y lo cierto es que la gente llega a los cursos con unas ideas ya adquiridas, que no se pueden cambiar con 4 ó 5 charlas. Aún así, creo que hay que aplicar las dos opciones, charlas específicas, y un trabajo cotidiano de reeducación.

En el colegio Sta. Teresa, que, como he comentado, cambia el profesorado todos los años, el director me ha pedido que el primer día que lleguen los nuevos maestros tenga una sesión con ellos para explicarles la realidad social del colegio, y acompañarles a conocer el barrio. El que se den cuenta de que eso es necesario desde un primer momento, creo que es ya un logro.

\section{V. ¿CÓMO SE TRABAJA ESTO?}

Digo cómo se trabaja, y no cómo se consigue, porque éste es un trabajo de largo plazo, donde se van cosechando pequeños éxitos y fracasos todos los días, en el que siempre se está aprendiendo y experimentando, y en el que el reto es no quemarte en los problemas diarios, sino tener un visión de largo plazo para poder valorar lo que se va consiguiendo, y al tiempo, ser capaz de transmitir esa visión optimista al resto de profesionales.

Hay que ser consciente desde un principio que los objetivos que te marcas en este trabajo no dependen exclusivamente de ti, sino que influyen otras muchas personas e instituciones y que, por tanto, tienes 
que ganarte la confianza y la colaboración de todas ellas para poder obtener resultados.

Yo lo tomo como un trabajo de comerciante, donde uno está siempre en contacto con la gente, y a cada uno ha de venderle un producto (a esto me ayuda el haber vendido en el mercado con mi padre):

- A las familias, la importancia que tiene para sus hijos el que saquen los estudios.

- A los colegios, el que son alumnos como cualquier otro, con los que se puede trabajar y obtener resultados, y que vale la pena apostar por ellos y contar con sus familias.

- A los alumnos el que ellos valen mucho, a nivel individual, y que pueden aprobar si se lo proponen, y que el colegio es mejor que estar en la calle.

Pero, para poder "vender estos productos" hay que tener en cuenta que la única arma con que se cuenta es con la credibilidad personal. El que lo respeten a uno, y que lo que esté proponiendo sea percibido como algo positivo para el interesado, ya que el monitor no tiene ninguna autoridad para imponerle nada a nadie. Uno debe de ganarse la confianza de cada uno de los sectores con los que trabaja (colegios, gabinete psicopedagógico, cada una de las familias extensas, alumnos, etc.), porque si no estableces previamente una situación de respeto mutuo, y de saber ponerte en la situación del otro, cuando quieras conseguir cosas no te van a hacer caso.

Cuando yo comencé a trabajar, me di cuenta que no podía llegar exigiendo cosas, ni a las familias, ni a los colegios. La estrategia fue el ganarme su confianza, y la forma que he utilizado es la de que antes de pedirle un favor a alguien ( $y$ se puede entender por un favor, el que no saquen a la niña del colegio con 12 años), tenías que haberle hecho tu varios favores a la familia. En este sentido, y teniendo en cuenta que mi trabajo no está definido por nadie, sino que lo diseño yo, suelo plantearlo no como una obligación por el sueldo que me pagan, sino como algo que podría no hacer pero que hago porque considero que es mejor para ellos.

Así, por ejemplo, cuando ayudo a las familias a rellenar las becas escolares, se lo planteo como algo que no tengo por qué hacer si no quiero, y las familias lo agradecen. Pero de la misma forma se lo planteo también al colegio, ya que si no les ayudo yo a rellenarlas tienen una avalancha de madres para que se las rellenen en el colegio, y si algún niño se queda sin echar la beca, cuando empieza el curso el tutor tiene problemas para que la familia pueda comprar los libros. No se trata de una cuestión de "me debes un favor", pero sí de "yo te ayudo 
cuando lo necesitas".

Muchas veces el ganarte la confianza es sencillamente saber escuchar a la gente, y que vean que eres capaz de entenderlo y de ponerte en su situación. Tanto en la situación de las familias gitanas, como en la situación de los profesores. Muchas veces los maestros se sienten frustrados como profesionales por no conseguir mejores resultados con los alumnos gitanos, y te cuentan sus problemas y necesitan que los entiendas, y después ellos están más predispuestos a aceptar tus consejos en el trato con los chavales o con las familias. Y lo mismo pasa con las familias.

Por tanto hay dos cuestiones fundamentales en este trabajo:

1. Tienes que ganarte a la gente con la que trabajas, ayudándoles en aquéllo que esté en tu mano, con el fin de que cuando tú pidas cosas haya cierta obligación moral de mirar por ti.

2. No puedes quedar mal con ninguna de las partes. Por mucho que te enfades no debes cerrarte puertas, porque en este trabajo vas a tener que relacionarte antes o después con la misma gente, aunque en ocasiones puedan entrarte ganas de "mandar a paseo" a una familia, o a un profesor, etc. Y esto no es siempre fácil, porque se dan situaciones complicadas, y encuentras profesores racistas o familias que te engañan..., pero es preferible evitar el enfrentamiento y buscar otras vías de solución, a quedar mal con un director y que se dificulte el trabajo con ese colegio.

\section{ESTRATEGIAS DE MEDIACIÓN}

$1^{\circ}$. Es fundamental ganarse la confianza de los directores de los colegios.

Ellos son la puerta de entrada para poder trabajar con los tutores. En este sentido hay que tener en cuenta: cómo se suele ver, de entrada, al monitor: "el defiende gitanos". La figura del monitor se percibe como un intruso que viene a vigilar los derechos de los gitanos frente a la actividad de los profesores, y a decirle al colegio lo que tiene que hacer. Como si no fuese suficiente con la desconfianza que de por sí les suponen los alumnos gitanos.

Mi estrategia fue intentar no responder a ese prejuicio, sino ofrecerme como alguien que viene a ayudar. $\mathrm{Y}$ ayudar suele suponer (desde la visión del colegio) solucionar problemas de comportamiento. En este sentido la forma fue echar una mano en la mediación con algunos chavales con los que tenían problemas, de la siguiente forma: por un 
lado, hablar con cada uno de los críos (la mayoría de los niños me respetan bastante, porque yo también los respeto a ellos, y porque tenemos una relación de mucho tiempo), e intentar marcarles objetivos de comportamiento asequibles (no el que se portasen bien, sino, pedirles como un favor personal el que durante un plazo limitado de tiempo no hiciesen cosas concretas de las que se quejaban los profesores). Y por otro lado, concerté una entrevista entre el director y el jefe de estudios y los padres de cada uno de estos críos. La actitud del director fue: "si quieres quedamos, pero no vendrán, llevamos dos años mandándoles citaciones y no han venido nunca". Pero, como a los padres fui a su casa a pedirles personalmente que fuesen a la reunión, y además yo me ofrecía a acompañarlos; pues sí que fueron. Las entrevistas fueron un tanto frías en el primer momento, pero cuando iban hablando la relación se estrechaba, y ambas partes (dirección y padres) se daban cuenta que "el otro" también estaba preocupado por el bien del chaval. El resultado fue una disminución (temporal, evidentemente) de los problemas diarios con esos niños, y un cambio de actitud del colegio hacia mi trabajo, ya que no había reaccionado justificando el comportamiento de los niños gitanos, y además, gracias a la mediación se había mejorado la situación escolar (para los directores, la mejora de la situación escolar de los niños gitanos, suele entenderse como la mejora del comportamiento. La mejora del aprendizaje es otra categoría, más cercana a los tutores).

Actualmente, en uno de los colegios tengo llave para entrar y salir cuando quiero, y en el otro, aunque no llego a tanto, lo cierto es que la relación es muy estrecha.

\section{$2^{\circ}$. Hay que ganarse la confianza de las familias gitanas.}

Me refiero a cada una de las familias gitanas extensas que residan en el municipio. Porque no sirve de nada tener una excelente relación con una familia, si no puedes acceder a otras.

Cuando comencé a trabajar, ya conocía a muchas de las familias gitanas, pero con algunas no tenía ningún trato. Además, cuando trabajas para los Servicios Sociales, te haces más "payo", aunque seas gitano (que no es mi caso). Quiero decir, que las familias te ven con cierto recelo, un poco como el espía de las asistentas sociales. Hay que decir que a mí me valieron mucho mis amistades previas con muchas familias gitanas, y también el haber trabajado con la mayoría de los niños, con los que tenía buena relación, algo que las familias valoran. Después el trato diario, el escuchar sus problemas y saber ponerte en su lugar, sin juzgarlos, y el ir haciendo distintos favores que no están directamente relacionados con mi trabajo (gestiones de papeles, dar la cara 
en alguna empresa para conseguir algún trabajo, etc.), ha hecho que la relación sea cada vez más cercana, y ha ido rompiendo los prejuicios que sé que algunas familias tenían sobre mí, tanto por ser "payo" como por trabajar para Servicios Sociales.

A la hora de trabajar con las familias, tengo claro que no pueden haber excepciones. Por un lado, es importante ganarse el respeto de aquellos miembros de las familias extensas que tienen un mayor peso dentro de la misma (independientemente de que tengan hijos en edad escolar o no), y por otro lado, también trabajo con las dos o tres familias que se dedican al tráfico de droga, ya que los objetivos escolares para sus hijos son los mismos que para el resto de niños y niñas.

\section{Ganarse la confianza y el respeto de los menores.}

Muchas veces, tanto el absentismo como el rendimiento escolar, dependen de la motivación que el niño o la niña tenga, y la posibilidad de motivarles para que apuesten por los estudios está en cierta medida condicionada por el cómo te vean a ti. El hecho de haber trabajado varios años como monitor de tiempo libre en los veranos con los niños y niñas gitanas, así como el haber sido monitor de comedor durante cinco cursos, hacía que los niños me conociesen, y existía una buena relación previa. Aún así, teniendo en cuenta que el trabajo de seguimiento escolar supone estar exigiéndoles cosas constantemente, he intentado compensarlo con actividades de ocio en fines de semana y en verano. Por ejemplo: llevarme a los niños a jugar al fútbol al polideportivo los sábados por la tarde; conseguirles entradas para el cine de la Casa de la Cultura cuando hay películas infantiles; llevármelos de excursión con la bici; ir a la piscina; etc. Estas actividades las voy condicionando en base a que se lo ganen. Por ejemplo, les digo: "el que no falte ningún día al colegio esta semana se viene el sábado al cine". Aunque en ocasiones tenga que bajar el listón con alguno para que se venga de vez en cuando.

Creo que el tener buena relación con los chavales facilita también la relación con los padres, ya que las familias valoran el trabajo que se hace con sus hijos.

\section{EL TRABAJO DÍA A DÍA}

\subsection{Protocolo municipal de absentismo escolar}

Hay que decir que el Ayuntamiento de Villena cuenta con un protocolo de absentismo, según el cual el orden de actuación en los casos de 
absentismo es el siguiente:

a) Cuando un tutor detecta que un niño falta a clase mucho (más de 5 días al mes), lo pone en conocimiento de la dirección del centro, que envía una citación a la familia recordándole que es obligatoria la asistencia, y que deben acudir al colegio para explicar la causa del absentismo, advirtiéndole que en caso de no acudir se dará parte a los Servicios Sociales.

b) En caso de que esa carta no surta efecto, se comunica a los Servicios Sociales, y éstos envían citación a la familia para que acuda a una entrevista con la trabajadora social, y explique el motivo del absentismo, recordándole en la misma su obligación como padres de responsabilizarse de que sus hijos acudan al colegio. Se le advierte que, en caso de que persista el absentismo, se hará un informe a la Comisión de Absentismo Escolar Municipal, y que ésta puede denunciar el caso a la Fiscalía de Menores para que tome las medidas oportunas.

c) Si la familia no acude a la cita, se la vuelve a citar, pero en esta segunda ocasión se envía a través de la policía municipal, que es quien la entrega en mano con acuse de recibo.

d) $\mathrm{Si}$, a pesar de todo esto, el absentismo persiste, la Comisión de Absentismo decide si se tramita una denuncia ante la Fiscalía de Menores (extremo al que nunca se ha llegado).

\subsection{La recogida de faltas en los colegios}

Si bien ésta es la forma teórica de actuar, considero que es una manera demasiado rígida, y además muy lenta, pues cuando el absentismo se ha consolidado es mucho más difícil motivar al menor para que acuda a gusto al colegio. Por ello, en el caso de los menores gitanos, que son con los que yo trabajo, el sistema es el siguiente:

Cuando comencé a trabajar, lo primero que hice fue presentarme a los directores de los colegios (acompañado por la trabajadora social que tuviese mejor relación con el centro), expresarle al director mi predisposición a colaborar con ellos para mejorar la escolaridad de los alumnos gitanos (no pedirles que ellos colaborasen con mi trabajo, para que no me viesen como un intruso, aunque en la práctica la colaboración es mutua), y solicitarles si podía ir al siguiente claustro del colegio para presentarme a todos los profesores, y explicarles cómo iba a plantear mi trabajo, y ponerme a su disposición para cualquier problema que tuviesen.

A partir de ahí, un día por semana acudo a cada uno de los colegios donde hay menores gitanos. Tengo un listado por cursos de todos los 
alumnos del colegio (gitanos y no gitanos), dividido por cuadrículas mensuales de faltas (cada tutor tiene también esas faltas). Previamente, he negociado con los directores de los colegios (y no ha sido fácil), el que cada tutor anote diariamente las faltas de los alumnos, diferenciando entre mañana y tarde. Es importante saber si las faltas son de mañana o tarde, porque un niño que suele faltar por las mañanas, es muy posible que tenga problemas en casa para despertarse y por tanto la solución hay que buscarla por ese camino, mientras que si las faltas suelen ser de tarde, la solución puede pasar por una beca de comedor escolar (si es porque los padres vienen tarde del mercado, o porque se queda durmiendo después de comer).

Como las faltas las recojo semanalmente, la relación con cada tutor es muy fluida, y al tiempo que recojo las faltas pregunto cómo le ha ido la semana con los distintos alumnos gitanos. Puedo hablar unos 5 minutos de media con cada tutor, pero es un tiempo suficiente para hacerme una idea de cómo le va a los chavales, y poder detectar problemas del tipo:

- Absentismo (obvio).

- Mal comportamiento (esto es algo que agobia mucho a los profesores, y en muchos casos es la función casi exclusiva para la que te quieren).

- Problemas de falta de material.

- Carencias relacionadas con los deberes.

- Necesidad de que estudie más en casa.

- O, a veces, la grata sorpresa de decirte que va bien o ha mejorado.

Recuerdo que al principio de comenzar a trabajar, un profesor que daba clases en $4^{\circ}$ de Primaria, no me facilitaba nada el trabajo y todo lo que hacía eran comentarios derrotistas y peyorativos sobre las posibilidades de estudio de los niños gitanos. Todo esto, poniéndome como prueba a un alumno gitano que tenía, y que en todo el curso no había conseguido que trajese (aunque fuesen sin hacer) las hojas de deberes que le mandaba, ni que trabajase en el aula. Estuve hablando con la madre, y quedamos en tratar, como mínimo, que bajase todos los días la cartera, y reforzarle los dos para que hiciese algo de deberes. En una semana el niño se acostumbró a subirse y bajarse la cartera de casa, y con parte de ayuda mía y el refuerzo de la madre comenzó a llevar los deberes hechos todos los días. A partir de ahí, en la entrevista con el tutor, éste me estuvo contando asombrado los progresos que había hecho en clase (a nivel de esfuerzo), y cambió su actitud con respecto a mi trabajo. Al mismo tiempo, los comentarios positivos sobre mi trabajo, que este profesor hizo con sus compañeros, sirvieron para mejorar 
la relación con otros profesores que en un principio eran escépticos ante la figura del monitor.

En estas entrevistas semanales, muchas veces me siento el psiquiatra de algunas maestras, porque muchas veces tienen problemas con alumnos gitanos y no entienden lo que pasa, o no saben cómo actuar, y necesitan comentarlo con alguien y que les entiendan. En este sentido, y a pesar de que muchas veces tienes que aguantar comentarios un tanto fuertes, el planteamiento que suelo tener es el de:

$1^{\circ}$ Decir que entiendo que es difícil en ocasiones, pero que está haciendo un gran trabajo.

$2^{\circ}$ Recordarles los logros que se han ido consiguiendo con los alumnos gitanos en los últimos años, y que no hay que quemarse en el día a día, sino tener una visión de largo plazo.

$3^{\text {o }}$ En algunos casos concretos, y confiando en su discreción, explicar los problemas de la familia, y el cómo pueden afectar a la situación que el niño vive en clase (esto suele servir para que se sitúen en la realidad que no ven, porque algunos profesores realmente desconocen que hay gente que vive en situaciones tercermundistas, o que tienen padres toxicómanos, etc.).

El día que estoy en cada colegio, aprovecho para tomar café en la sala de profesores a la hora del recreo, y en cierto modo, llegas a ser visto casi como un compañero por parte de muchos maestros, lo que facilita el trabajo.

\subsection{El trabajo con las familias}

Una vez que conozco las faltas de asistencia, me paso por las casas a hablar con las familias. Intento ir entre lunes y miércoles, porque el efecto de la negociación con las familias suele tener una duración limitada en el tiempo (depende de casos), y si voy un jueves o un viernes, cabe la posibilidad de que acudan a clase un día, llegue el fin de semana, y dejen de ir el lunes. Mi experiencia me dice que en muchos casos el acudir a clase es una cuestión de inercia, cuando se coge el hábito de ir todos los días no se falta, y cuando faltan (por cualquier cuestión) varios días, se acostumbran a no ir. Así, que prefiero hablar con la familia a primeros de semana, y que cojan la inercia de ir toda la semana.

Cuando voy a las casas nunca planteo la cuestión de las faltas directamente, sino que suelo comentar que he estado en el colegio y que me han dicho como iba el niño, y que era una lástima porque siendo tan listo (o tan bueno, o cualquier cosa positiva) que al faltar pues se está quedando descolgado de sus compañeros (suelo buscar comparacio- 
nes con algún otro niño gitano que vaya bien, a fin de que sirva de referente para la familia, y no lo vean como una cosa de payos). Intento que lo vean como que estoy allí, no para exigirles que lleven más a su hijo al colegio, sino porque me preocupo por el chaval y quiero lo mejor para el.

Las visitas pueden ser por el tema de absentismo, pero también por cuestiones relacionadas con los deberes, con que se acerque al colegio a las 12 para que el maestro le explique como va el nene, etc.

Cuando se trata de una familia con la que creo que la negociación puede ser más dura, o que pueden estar menos predispuestos con el tema escolar, en ocasiones utilizo la siguiente estrategia: Unos días antes me paso por la casa con alguna excusa que suponga un favor por mi parte (algún favor que me hayan pedido que les mire, o llevarles una foto que tenía del hijo, o de una boda, etc.), y charlo un poco con ellos sin hacer ninguna referencia al tema escolar. De esta forma, cuando voy unos días más tarde a hablarles del tema del colegio (como algo que me acaban de comentar), la predisposición de la familia es mucho mejor.

Si una familia, a pesar de hablar con ellos, no consigo que vaya el niño al colegio, analizo quién es la persona de su familia que pueda tener más influencia sobre ellos. En ocasiones es el marido (porque en algunas casas el marido se va a trabajar todo el día, y no sabe que sus hijos faltan al colegio), y vas por la noche y todo se soluciona. En otros casos (la mayoría), es el abuelo paterno (de ahí mi hincapié en ganarme a los gitanos viejos), otras el hermano mayor, o incluso una hermana de la madre, (el tener claras las relaciones familiares existentes, es fundamental para desarrollar el trabajo). En este caso me acerco a la casa de esta persona, y le explico que tengo un problema, que no es un favor para mí, pero que es una lástima que los niños no vayan al colegio, y que los padres no se preocupan. En resultado suele ser que te digan: "no te preocupes que yo hablaré con el padre", y suele tener efecto.

En caso de que nada de esto funcione, les explico que a mí me pagan lo mismo vayan o no, que es por el niño, y que mi trabajo es recoger las faltas de los niños en los colegios y entregarlas en Servicios Sociales. Que les he estado tapando durante un tiempo, pero que no me puedo jugar mi trabajo porque es el pan de mi hijo, y que si no lo llevan al colegio yo tengo que dar parte, que no lo tomen a mal, y que lo que pase después ya no es responsabilidad mía.

El año pasado tuve dificultades con una familia en la que uno de sus hijos de 10 años, no iba nunca al colegio. El niño se negaba, y su padre 
que es toxicómano no se imponía. La familia cobraba una ayuda social, con la que comían, y si yo lo comunicaba a Servicios Sociales la iban a perder. Los intentos con los padres habían sido infructuosos, así que fui a hablar con el abuelo (el hombre me estima mucho, entre otras cosas porque ve que me mareo por sus nietos). Le expliqué la situación, y el hombre me pidió como un favor que le dejase unos días para que él hablase con su hijo, y que si a la semana siguiente seguía faltando su nieto, que lo comunicase a donde hiciese falta, que él lo entendía.

En algunos casos, una familia me comenta que necesitan que el niño o la niña falte al colegio algunos días por cualquier motivo (normalmente por cuestiones laborales). En estos casos negocio con ellos una fecha concreta para que vuelvan al colegio, y durante esos días yo no comunico a nadie las faltas. Esto tiene normalmente buenos resultados, ya que si no fuese negociado, faltarían de igual forma, y seguramente no volverían a ir al colegio.

\subsection{El programa despertador}

Al poco tiempo de estar trabajando como monitor, tuve un caso de un niño de 9 años que faltaba al colegio porque vivía sólo con su padre (en el barrio del Rabal), y cuando el padre se iba a trabajar el niño se quedaba durmiendo. El padre me pidió como un favor el que fuese por la mañana a despertar al niño como única forma de que no faltase al colegio.

A partir de subir todas las mañanas al barrio, cuando tenía algún caso de un niño que faltaba porque la madre no podía bajarlo por las mañanas (en un primer momento fueron dos madres que tenían un niño recién nacido y otro de 4 ó 5 años), le ofrecía el favor de llevármelos también al colegio, ya que de todas formas tenía que ir con el otro niño. Las madres accedieron contentas y muy agradecidas de mi preocupación por los críos. (Hay que decir que los llevo al colegio, pero que nunca los recojo).

El ir al barrio por las mañanas supuso que muchos niños que antes bajaban solos, me esperaban y se venían conmigo. De manera que hay días que bajo rodeado de 15 ó 20 niños. Esto me ha servido de excusa para acercarme a las casas de algunos de esos niños (concretamente a las casas donde se suelen quedar durmiendo los padres y faltan al colegio), y llamar para preguntarles si se viene el hijo conmigo.

El efecto que ha tenido este programa sobre el absentismo ha sido importantísimo, ya que niños que faltaban casi todas las mañanas, ahora casi no faltan. Pero además de la reducción del absentismo escolar, tiene otras ventajas: 
- Me permite una relación diaria con las familias, de manera que al tiempo que voy a las casas a despertar o recoger a los niños, me informan de cosas o bien yo les doy recados de los maestros, o sencillamente hablamos de cualquier cosa que puede no tener nada que ver con la escolaridad pero que sirve para mantener una buena relación personal.

- La imagen del monitor por parte de las familias es una imagen positiva. Ven que no sólo les exiges que vayan al colegio sus hijos, sino que te molestas en subir a las casas a levantarlos si hace falta, o llevarlos en tu coche si llueve, y por tanto que no es un trabajo que haces sencillamente por que te pagan, sino porque aprecias a sus hijos. (Dejo siempre claro a las familias que el subir a por los niños lo hago porque quiero, que no es algo que me mande el Ayuntamiento).

- Me permite preguntarles a los niños por la mañana si han hecho los deberes (algunos me los enseñan), y si llevan todo el material que necesitan para ese día. Así, algunos profesores que se quejaban de niños concretos que nunca llevaban el material que tocaba, ahora están sorprendidos del cambio en los niños, y hay algún crío que hace los deberes casi con la ilusión de enseñármelos a mí por la mañana.

Como contraposición, este programa tiene el riesgo de que algunas familias se acomoden a que les despierten y les lleven los niños al colegio, y por tanto que suponga una menor implicación en sus obligaciones hacia la escolaridad de sus hijos (esto siempre me lo están echando en cara los profesores). Creo que hay alguna familia que sí le pueda estar pasando esto (en estos casos lo que hago es no pasar de vez en cuando por su casa, para que sepan que no se pueden confiar totalmente conmigo, porque si no puedo ir no tengo por qué hacerlo), pero también considero que hay algunos niños que por sus circunstancias familiares, no irían ninguna mañana al colegio si no los recogiese.

\section{A MODO DE CONCLUSIÓN}

Lo que he expuesto aquí son simplemente los objetivos que en mi trabajo como mediador (en Villena se le llama monitor, aunque las funciones sean las mismas) me marco, y a grandes rasgos cómo desarrollo ese trabajo en el día a día. No quiero decir con esto que sea la mejor forma de trabajar, ni que sea extrapolable a cualquier sitio. Sencillamente es una experiencia concreta en un municipio concreto.

No he hablado de resultados, pero no quisiera dar la imagen de que 
todo es perfecto, ni de que todos los objetivos que me marco los consigo al $100 \%$. El día a día va dejando pequeños éxitos y pequeños fracasos, y mucho desgaste personal. Se trata de un trabajo a largo plazo, como una carrera de fondo, y la única forma que tengo de no quemarme es ir agarrándome a los pequeños logros que se van consiguiendo (por ejemplo, que un chaval llegue a la Universidad), y analizar los resultados comparativamente con años anteriores, para poder apreciar que se va progresando, y que el esfuerzo diario vale la pena. 